\title{
THE GENERAL CHINESE REMAINDER THEOREM
}

\author{
Sorin Iftene ${ }^{1)}$, Florin Chelaru ${ }^{2)}$ \\ ${ }^{1)}$ Faculty of Computer Science, "Al. I. Cuza" University, Iasi, Romania \\ e-mail: siftene@infoiasi.ro \\ ${ }^{2)}$ Faculty of Computer Science, "Al. I. Cuza" University, Iasi, Romania \\ e-mail: florin.chelaru@infoiasi.ro
}

\begin{abstract}
The Chinese remainder theorem deals with systems of modular equations. The classical variant requires the modules to be pairwise coprime. In this paper we discuss the general variant, which does not require this restriction on modules. We have selected and implemented several algorithms for the general Chinese remainder theorem. Moreover, we point out some interesting applications of this variant in secret sharing and threshold cryptography.
\end{abstract}

Keywords: The Chinese remainder theorem, secret sharing, threshold cryptography.

\section{INTRODUCTION AND PRELIMINARIES}

The Chinese remainder theorem deals with systems of modular equations. It has many applications in computer science (see, for example, [1]). We only mention the RSA decryption algorithm proposed by Quisquater and Couvreur [2], the discrete logarithm algorithm proposed by Pohlig and Hellman [3] and the algorithm of recovering the secret in the Mignotte's threshold secret sharing scheme [4] or in the Asmuth-Bloom threshold secret sharing scheme [5]. The classical variant requires that modules be pairwise coprime. In this paper we discuss the general variant, which does not require this restriction on modules.

The paper is organized as follows. The rest of this section is dedicated to some preliminaries on number theory, focusing on the Chinese remainder theorem. We survey the most important algorithms for the general Chinese remainder theorem in Section 2. Implementation details and test results are discussed in the next section. Some interesting applications of the general variant of the Chinese remainder theorem are presented in Section 4. Our conclusions are presented in the last section.

We present next some basic facts on number theory. For more details, the reader is referred to [6] and computational aspects can be found in [7].

Let $a, b \in \mathbf{Z}, b \neq 0$. The quotient of the integer division of $a$ by $b$ will be denoted by $a \operatorname{div} b$ and the remainder will be denoted by $a \bmod b$. Moreover, in case that $a \bmod b=0$ we shall say that $b$ is a divisor of $a$ or $b$ divides $a$ and we shall use the notation $b \mid a$.

$$
\text { Let } \quad a_{1}, \ldots, a_{n} \in \mathrm{Z}, a_{1}^{2}+\cdots+a_{n}^{2} \neq 0 . \quad \text { The }
$$
greatest common divisor ( $\mathrm{gcd}$ ) of $a_{1}, \ldots, a_{n}$ will be denoted by $\left(a_{1}, \ldots, a_{n}\right)$. We say that $a_{1}, \ldots, a_{n}$ are coprime if $\left(a_{1}, \ldots, a_{n}\right)=1$.It is well-known that there exist $\alpha_{1}, \ldots \alpha_{n} \in \mathbf{Z}$ which satisfy

$$
\alpha_{1} a_{1}+\cdots+\alpha_{n} a_{n}=\left(a_{1}, \ldots, a_{n}\right)
$$

(the linear form of the gcd).

Let $a_{1}, \ldots, a_{n} \in \mathbf{Z}$ such that $a_{1} \cdots a_{n} \neq 0$. The least common multiple $(\mathrm{lcm})$ of $a_{1}, \ldots, a_{n}$ will be denoted by $\left[a_{1}, \ldots, a_{n}\right]$.

Let $a, b, m \in \mathbf{Z}$. We say that $a$ and $b$ are congruent modulo $m$, and we use the notation $a \equiv b \bmod m \quad$ or $\quad a \equiv_{m} b, \quad$ if $m \mid(a-b) . \quad \mathbf{Z}_{m}$ denotes the set $\{0, \ldots, m-1\}$ for any $m \geq 2$.

We shall present next the general variant of the Chinese remainder theorem:

Theorem 1. ([8]) The system of equations

$$
\left\{\begin{array}{c}
x \equiv b_{1} \bmod m_{1} \\
\vdots \\
x \equiv b_{k} \bmod m_{k}
\end{array}\right.
$$

has solutions in $\mathbf{Z}$ if and only if

$$
b_{i} \equiv b_{j} \bmod \left(m_{i}, m_{j}\right)
$$

for all $1 \leq i, j \leq k$. Moreover, if the above system of equations has solutions in $\mathbf{Z}$, then it has an 
unique solution in $\mathrm{Z}_{\left[m_{1}, \ldots, m_{k}\right]}$.

The direct implication can be easily proven as follows. Assume first that $x_{0}$ is an integer solution of the system (1) and let $1 \leq i, j \leq k$. The relation $x \equiv b_{i} \bmod m_{i}$ implies that $x \equiv b_{i} \bmod \left(m_{i}, m_{j}\right)$. Moreover, by the same argument, we have also $x \equiv b_{j} \bmod \left(m_{i}, m_{j}\right)$ which leads, by transitivity, to $b_{i} \equiv b_{j} \bmod \left(m_{i}, m_{j}\right)$.

The uniqueness modulo $\left[m_{1}, \ldots, m_{k}\right]$ can also be easily proven. Let $x_{0}$ and $y_{0}$ be two solutions for the system (1). By transitivity we obtain that $x_{0} \equiv y_{0} \bmod m_{i}$, for all $1 \leq i \leq k$, which implies that $\left(x_{0}-y_{0}\right)$ is a multiple of $m_{1}, \ldots, m_{k}$, which leads to $\left[m_{1}, \ldots, m_{k}\right] \mid\left(x_{0}-y_{0}\right)$.

The converse implication will be discussed in Section 2.

An important particular case is the case $\left(m_{i}, m_{j}\right)=1$ for all $1 \leq i<j \leq k$. In this case, for any integers $b_{1}, \ldots, b_{k}$, the system of equations

$$
\left\{\begin{array}{c}
x \equiv b_{1} \bmod m_{1} \\
\vdots \\
x \equiv b_{k} \bmod m_{k}
\end{array}\right.
$$

has an unique solution in $\mathbf{Z}_{m_{1} \cdots m_{k}}$.

\section{ALGORITHMS FOR THE GENERAL CHINESE REMAINDER THEOREM}

In this section we will present the most important algorithms for the general variant of the Chinese remainder theorem.

\subsection{ORE'S ALGORITHM}

Ore [8] has proposed an interesting proof for the general Chinese remainder theorem.

Let $m=\left[m_{1}, \ldots, m_{k}\right]$ and $c_{i}=\frac{m}{m_{i}}$, for $1 \leq i \leq k$.

Claim 1: $\left(c_{1}, \ldots, c_{k}\right)=1$.

We first choose

$$
D=\left\{p \text { prime } \mid(\exists 1 \leq i \leq k)\left(p \mid m_{i}\right)\right\} .
$$

Thus, every element $m_{i}$ can be written as

$$
m_{i}=\prod_{p \in D} p^{e_{p, i}}
$$

(with $e_{p, i}=0$ if $p$ does not divide $m_{i}$ ), and

$$
\begin{gathered}
{\left[m_{1}, \ldots, m_{k}\right]=\prod_{p \in D} p^{\max \left(e_{p, 1}, \ldots, e_{p, k}\right)},} \\
c_{i}=\prod_{p \in D} p^{\max \left(e_{p, 1}, \ldots, e_{p, k}\right)-e_{p, i}} .
\end{gathered}
$$

For every $p \in D$, there is an $i, 1 \leq i \leq k$ such that $e_{p, i}=\max \left(e_{p, 1}, \ldots, e_{p, k}\right)$. In this case, $p$ does not divide $c_{i}$ which implies that for every possible common prime divisor $p$ there is an element $i, 1 \leq i \leq k$, such that $p$ does not divide $c_{i}$ which eventually leads to $\left(c_{1}, \ldots, c_{k}\right)=1$.

Thus, there are $\alpha_{1}, \ldots, \alpha_{k} \in \mathbf{Z}$ that satisfy $\alpha_{1} c_{1}+\cdots+\alpha_{k} c_{k}=1$.

Claim 2: $\frac{m_{i}}{\left(m_{i}, m_{j}\right)} \mid c_{j}$, for all $1 \leq i, j \leq k$.

We have that

$$
\frac{m_{i}}{\left(m_{i}, m_{j}\right)}=\prod_{p \in D} p^{e_{p, i}-\min \left(e_{p, i}, e_{p, j}\right)}
$$

and so, the relation $\frac{m_{i}}{\left(m_{i}, m_{j}\right)} \mid c_{j}$ is equivalent to $e_{p, i}-\min \left(e_{p, i}, e_{p, j}\right) \leq \max \left(e_{p, 1}, \ldots, e_{p, k}\right)-e_{p, j}$ for every $p \in D$, which clearly holds true.

Claim 3: The value

$$
x_{0}=\left(\alpha_{1} c_{1} b_{1}+\cdots+\alpha_{k} c_{k} b_{k}\right) \bmod m
$$

is a solution of the system of equations (1).

Let $i$ be an arbitrary element, $1 \leq i \leq k$. For every $1 \leq j \leq k$ we have

$$
\begin{aligned}
b_{j} \equiv_{\left(m_{i}, m_{j}\right)} b_{i} & \Rightarrow c_{j} b_{j} \equiv_{c_{j}\left(m_{i}, m_{j}\right)} c_{j} b_{i} \\
& \Rightarrow c_{j} b_{j} \equiv_{m_{i}} c_{j} b_{i} \quad(\text { by Claim 2) } \\
& \Rightarrow \alpha_{j} c_{j} b_{j} \equiv_{m_{i}} \alpha_{j} c_{j} b_{i}
\end{aligned}
$$

If we sum the last congruencies, for $1 \leq j \leq k$, we obtain

$$
\alpha_{1} c_{1} b_{1}+\cdots+\alpha_{k} c_{k} b_{k} \equiv_{m_{i}} b_{i}\left(\alpha_{1} c_{1}+\cdots+\alpha_{k} c_{k}\right)
$$

which finally leads to $x_{0} \equiv b_{i} \bmod m_{i}$.

This demonstration leads to Ore's algorithm:

CRT_Ore $\left(b_{1}, \ldots, b_{k}, m_{1}, \ldots, m_{k}\right)$

Input: $b_{1}, \ldots, b_{k}, m_{1}, \ldots, m_{k} \in \mathbf{Z}$ such that

$b_{i} \equiv b_{j} \bmod \left(m_{i}, m_{j}\right), \forall 1 \leq i<j \leq k$;

Output: $x_{0}$, the unique solution 
modulo $\left[m_{1}, \ldots, m_{k}\right]$ for the system (1);

\section{begin}

1.

$$
m=\left[m_{1}, \ldots, m_{k}\right] \text {; }
$$

2. for $i:=1$ to $k$ do $c_{i}=\frac{m}{m_{i}}$;

3. find $\alpha_{1}, \ldots, \alpha_{k} \in \mathrm{Z}$ such that

4. $\alpha_{1} c_{1}+\cdots+\alpha_{k} c_{k}=1$;

$$
x_{0}=\sum_{i=1}^{n} \alpha_{i} \cdot c_{i} \cdot b_{i} \bmod m \text {; }
$$

end.

Gauss [9] has described a similar algorithm for the case of pairwise coprime modules.

\subsection{FRAENKEL'S ALGORITHM}

We have also rediscovered a more interesting algorithm for the general Chinese remainder theorem, algorithm presented by Fraenkel in [10]. The idea is to consider $x_{i}$, the unique solution modulo $\left[m_{1}, \ldots, m_{i}\right]$ of the system of equations

$$
\left\{\begin{array}{l}
x \equiv b_{1} \bmod m_{1} \\
\vdots \\
x \equiv b_{i} \bmod m_{i}
\end{array}\right.
$$

for all $1 \leq i \leq k$. Thus, we have that $x_{1}=b_{1} \bmod m_{1}$ and that $x_{k}$ is the required solution.

Consider now $1 \leq i \leq k-1$. The system of equations

$$
\left\{\begin{array}{l}
x \equiv x_{i} \bmod \left[m_{1}, \ldots, m_{i}\right] \\
x \equiv b_{i+1} \bmod m_{i+1}
\end{array}\right.
$$

has an unique solution in $\mathrm{Z}_{\left[\left[m_{1}, \ldots, m_{i}\right], m_{i+1}\right]}=\mathrm{Z}_{\left[m_{1}, \ldots, m_{i+1}\right]}$, namely $x_{i+1}$. From the first equation, $x_{i+1}$ must be of form $x_{i}+y \cdot\left[m_{1}, \ldots, m_{i}\right]$, for some $y \in \mathbf{Z}$. By replacing $x$ using this form in the second equation, we get the equation

$$
x_{i}+y \cdot\left[m_{1}, \ldots, m_{i}\right] \equiv b_{i+1} \bmod m_{i+1} .
$$

From this equation, we have

$$
y=\frac{b_{i+1}-x}{\left(c_{i}, m_{i+1}\right)} \cdot\left(\frac{c_{i}}{\left(c_{i}, m_{i+1}\right)}\right)^{-1} \bmod \frac{m_{i+1}}{\left(c_{i}, m_{i+1}\right)},
$$

where $c_{i}=\left[m_{1}, \ldots, m_{i}\right]$, for all $1 \leq i \leq k$.

We obtain the next algorithm:

$$
\text { CRT_Fraenkel }\left(b_{1}, \ldots, b_{k}, m_{1}, \ldots, m_{k}\right)
$$

Input: $b_{1}, \ldots, b_{k}, m_{1}, \ldots, m_{k} \in \mathbf{Z}$ such that

$b_{i} \equiv b_{j} \bmod \left(m_{i}, m_{j}\right), \forall 1 \leq i<j \leq k$;

Output: $x_{0}$, the unique solution modulo $\left[m_{1}, \ldots, m_{k}\right]$ for the system (1);

begin

1. for $i:=1$ to $k-1$ do $c_{i}:=\left[m_{1}, \ldots, m_{i}\right]$;

2. $\quad x_{0}:=b_{1} \bmod m_{1}$;

3. for $i:=1$ to $k-1$ do

begin

4. $y:=\frac{b_{i+1}-x}{\left(c_{i}, m_{i+1}\right)} \cdot\left(\frac{c_{i}}{\left(c_{i}, m_{i+1}\right)}\right)^{-1} \bmod \frac{m_{i+1}}{\left(c_{i}, m_{i+1}\right)} ;$

5.

$x:=x+y \cdot c_{i}$

end

end.

When $\left(m_{i}, m_{j}\right)=1$, for all $1 \leq i<j \leq k$, we obtain Garner's algorithm [11].

\subsection{ALGORITHMS BASED ON THE PRIME FACTORIZATIONS OF THE MODULES}

Suppose that we know all the prime factors of the involved modules and that these are $p_{1}, \ldots, p_{l}$. In this case, every module can be written as

$$
m_{i}=\prod_{j=1}^{l} p_{j}^{e_{i, j}}
$$

for all $1 \leq i \leq k$, with $e_{i, j}=0$ in case that $p_{j}$ does not divide $m_{i}$. In this case, the equation $x \equiv b_{i} \bmod m_{i}$ is equivalent to the system of modular equations $x \equiv b_{i} \bmod p_{j}^{e_{i, j}}, 1 \leq j \leq l$.

Consider $e_{j}=\max \left(\left\{e_{i, j} \mid 1 \leq i \leq k\right\}\right)$, for all $1 \leq j \leq l$, and an index $i_{j}, 1 \leq i_{j} \leq k$, such that $e_{j}=e_{i_{j}, j}$. In this case, the system (1) can be rewritten as

$$
\left\{\begin{array}{c}
x \equiv b_{i_{1}} \bmod p_{1}^{e_{1}} \\
\vdots \\
x \equiv b_{i_{l}} \bmod p_{l}^{e_{l}}
\end{array}\right.
$$

Indeed, because the knowledge of the remainder of integer division of a positive integer $a$ by a prime 
power $p^{e}$ implies the knowledge of the remainder of integer division of $a$ by any $p^{e^{\prime}}$ with $1 \leq e^{\prime} \leq e-1$, it is sufficient to consider only the equations with the greatest prime powers. In this way, any system of modular equations can be reduced to one with pairwise coprime modules. Garner's algorithm or Gauss' algorithm can be used for solving the system (2).

\section{IMPLEMENTATION DETAILS AND TEST RESULTS}

\subsection{IMPLEMENTATION DETAILS}

The large number operations and algorithms are inspired from [7] and have been considered for the only purpose of comparing the two algorithms (Ore's and Fraenkel's). Thus, only few improvements have been made in order to achieve certain performance.

Addition and subtraction. We have used the classical algorithms for these two operations. There is no need of getting into details at this part.

Multiplication. For few digits numbers we have used the classical School Multiplication, while for large amounts of digits, we chose Karatsuba Multiplication, which provided a certain speed improvement.

Division and modular reduction. Speed related reasons led us to choose Recursive Division for numbers with many digits, leaving the smaller ones to the classical School Division.

The (extended) greatest common divisor. We stopped upon the (extended) Binary Algorithm .

The least common multiple. We used a simple algorithm for this particular operation: for two numbers, we made use of the fact that

$$
[a, b]=\frac{a \cdot b}{(a, b)}
$$

and for more numbers, we split them into two groups, for which we computed recursively the $l \mathrm{~cm}$. Then, with the results, we applied the above formula.

We designed the library in Microsoft ${ }^{\circledR}$ Visual $\mathrm{C}++{ }^{\circledR} 6.0$ and it is only compatible with Windows ${ }^{\circledR}$ operating systems. The test system was an AMD Athlon $^{\mathrm{TM}}$ XP 2700+ with 1024 MB RAM.

\subsection{COMPARISONS BETWEEN ORE'S AND FRAENKEL'S ALGORITHM}

We have considered systems with 5 equations and tested the two algorithms for modules with sizes up to 2048 bits. We divided the comparisons into two sections, which we considered to be distinct, and yet, each very important.

First of them is the comparison for the case that modules are not known in advance.

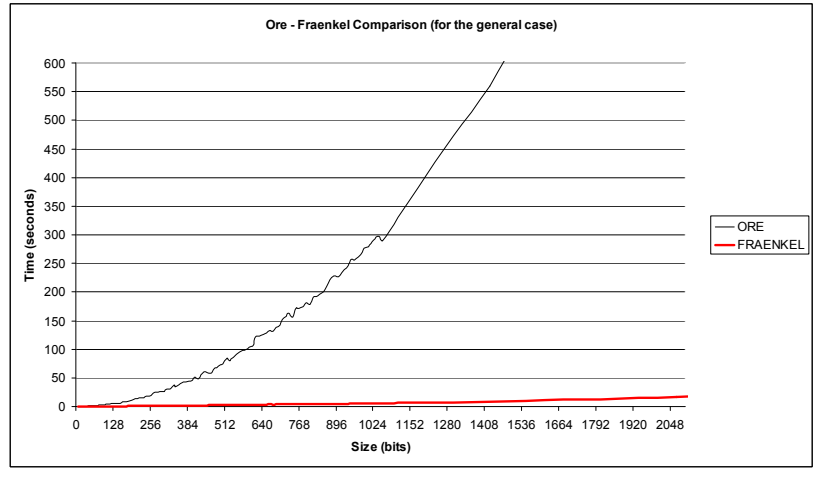

It is clear that Fraenkel's algorithm behaves much better than Ore's algorithm. This can be motivated by the fact that finding $\alpha_{1}, \ldots, \alpha_{k} \in \mathrm{Z}$ such that $\alpha_{1} c_{1}+\cdots+\alpha_{k} c_{k}=1$ (Step 3 from Ore's algorithm) requires a lot of time.

The second one assumes that modules are known in advance and, thus, some pre-computations can be performed.

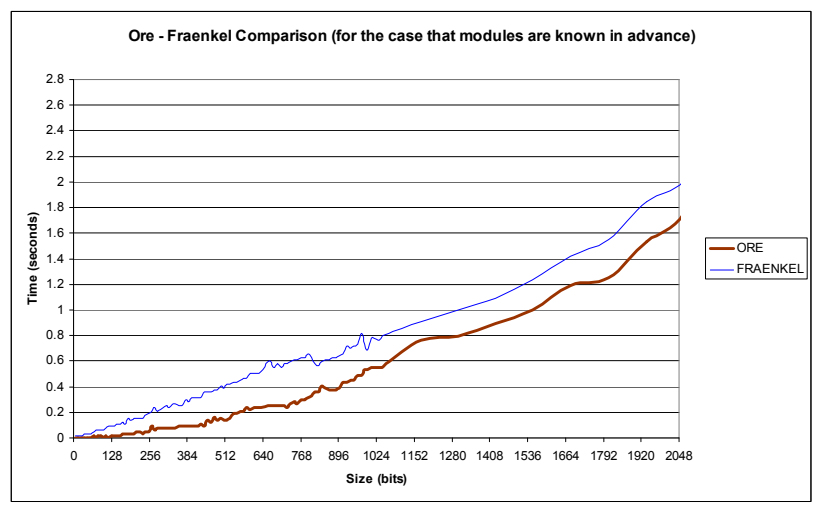

We have excluded the time for these precomputations (Steps 1, 2, 3 for Ore's algorithm and the computations of values $c_{i},\left(c_{i}, m_{i+1}\right)$ and the other related values in Fraenkel's algorithm).

In this case, Ore's algorithm behaves better than Fraenkel's algorithm. This can be motivated by the fact that Fraenkel's algorithm has two steps in which pre-computation can not be entirely done (Steps 4, 5) as opposed to Ore's algorithm.

\section{APPLICATIONS IN CRYPTOGRAPHY}

In this section we point out some interesting applications of the general variant of the Chinese remainder theorem. More exactly, we will discuss a generalization of Mignotte's threshold secret sharing scheme and its application to threshold 
cryptography.

\subsection{SECRET SHARING}

A secret sharing scheme starts with a secret and then derives from it certain shares (or shadows) such that the secret may be recovered only in the case of possessing a certain predetermined set of shares. The initial applications of secret sharing were safeguarding cryptographic keys and providing shared access to strategical resources. Threshold cryptography (see, for example, [12]) and some evoting schemes (see, for example, [13]) are some examples of more recent applications of the secret sharing schemes.

In the first secret sharing schemes only the cardinality of the sets of shares was important for recovering the secret. Such schemes have been referred to as threshold secret sharing schemes. We mention Shamir's threshold secret sharing scheme [14] based on polynomial interpolation, Blakley's geometric threshold secret sharing scheme [15], Mignotte's threshold secret sharing scheme [4] and Asmuth-Bloom threshold secret sharing scheme [5], both based on the Chinese remainder theorem. Ito, Saito, and Nishizeki [16], Benaloh and Leichter [17] give constructions for more general secret sharing schemes.

Let $n$ be an integer, $2 \leq n$ and $\Pi$ a set of subsets of $\{1,2, \ldots, n\}$. Informally, a $\Pi$-secret sharing scheme is a method of generating the elements $\left(S,\left(I_{1}, \ldots, I_{n}\right)\right)$ such that

(1) for all $A \in \Pi$, the problem of finding the element $S$, given the set $\left\{I_{i} \mid i \in A\right\}$, is "easy";

(2) for all $A \notin \Pi$, the problem of finding the element $S$, given the set $\left\{I_{i} \mid i \in A\right\}$, is intractable.

The set $\Pi$ will be referred to as the authorized access structure or simply as the access structure, $S$ will be referred to as the secret and $I_{1}, \ldots, I_{n}$ will be referred to as the shares (or the shadows) of $S$. The elements of the set $\Pi$ will be referred to as the authorized access sets of the scheme.

A natural condition is that an access structure $\Pi$ is monotone, i.e.,

$(\forall B \subseteq\{1,2, \ldots, n\})((\exists A \in \Pi)(A \subseteq B) \Rightarrow B \in \Pi)$

In this case, the access structure $\Pi$ is well specified by the set of the minimal authorized access sets, i.e., the set

$$
\Pi_{\text {min }}=\{A \in \Pi \mid(\forall B \in \Pi-\{A\})(\neg B \subseteq A)\} .
$$

In case $\Pi_{\min }=\{A \subseteq\{1,2, \ldots n\} \| \mathrm{A} \mid=\mathrm{k}\}$, for some positive integer $k, 2 \leq k \leq n$, a $\Pi$-secret sharing scheme will be referred to as an $(k, n)$ threshold secret sharing scheme.
In [18] we have proposed a generalization of Mignotte's threshold secret sharing scheme. Our scheme is based on some particular sequences of integers, namely the generalized Mignotte sequences. More exactly, a generalized ( $k, n)$ Mignotte sequence is a sequence of $n$ positive integers such that

$$
\max _{1 \leq i_{1}<\cdots<i_{k-1} \leq n}\left(\left[m_{i_{1}}, \ldots, m_{i_{k-1}}\right]\right)<\min _{1 \leq i_{1}<\cdots<i_{k} \leq n}\left(\left[m_{i_{1}}, \ldots, m_{i_{k}}\right]\right)
$$

Having a publicly known generalized $(k, n)$ Mignotte sequence $m_{1}, \ldots, m_{n}$, the scheme continues as follows:

- The secret $S$ is chosen as a random integer with $\beta<S<\alpha, \quad \beta=\max _{1 \leq i_{1}<\cdots<i_{k-1} \leq n}\left(\left[m_{i_{1}}, \ldots, m_{i_{k-1}}\right]\right)$

and $\alpha=\min _{1 \leq i_{1}<\cdots<i_{k} \leq n}\left(\left[m_{i_{1}}, \ldots, m_{i_{k}}\right]\right)$;

- The shares $I_{1}, \ldots, I_{n}$ are chosen as follows:

$$
I_{i}=S \bmod m_{i}
$$

for all $1 \leq i \leq n$;

- Having the shares $I_{i_{1}}, \ldots, I_{i_{k}}$, the secret $S$ can be obtained, using the general variant of the Chinese remainder theorem, as the unique solution modulo $\left[m_{i_{1}}, \ldots, m_{i_{k}}\right]$ of the system

$$
\left\{\begin{aligned}
x & \equiv I_{i_{1}} \bmod m_{i_{1}} \\
& \vdots \\
x & \equiv I_{i_{k}} \bmod m_{i_{k}}
\end{aligned}\right.
$$

For $\left(m_{i}, m_{j}\right)=1$, for all $1 \leq i<j \leq n$, we obtain Mignotte's threshold secret sharing scheme [4].

\subsection{THRESHOLD CRYPTOGRAPHY}

In threshold (or group-oriented) cryptography (see, for example, [12]), the capacity of performing cryptographic operations such as decryption or digital signature generation is shared among members of a certain group. This can be achieved by combining multiplicative secret sharing schemes [19] with homomorphic cryptographic . operations. The majority of the threshold cryptographic schemes are based on Shamir's secret sharing scheme. In [20] we have indicated how to combine the threshold secret sharing schemes based on the general Chinese remainder theorem with $R S A$ cryptosystem [21] in order to get threshold decryption or signature generation. To be precise, the value $x^{d} \bmod N$ must be collectively computed by any $k$ of $n$ users, where $N=p q$, with 
$p$ and $q$ primes, and $(d,[p-1, q-1])=1$.

We will briefly present this scheme (for more details, the reader is referred to [20]). The dealer chooses a generalized Mignotte sequence $m_{1}, \ldots, m_{n}$ such that $[p-1, q-1] \mid m_{i}, \quad$ for all $1 \leq i \leq n$ and $\beta<d<\alpha$. The dealer computes the shares $I_{1}, \ldots, I_{n}$ as above and distributes them securely to users. By Ore's construction, the secret key $d$ can be expressed as

$$
d=\left(\alpha_{i_{1}} c_{i_{1}} I_{i_{1}}+\cdots+\alpha_{i_{k}} c_{i_{k}} I_{i_{k}}\right) \bmod m_{i_{1}} \cdots m_{i_{k}},
$$

for any set $\left\{i_{1}, \ldots, i_{k}\right\} \subseteq\{1,2, \ldots n\}$, where $c_{i_{j}}=\frac{m_{i_{1}} \cdots m_{i_{k}}}{m_{i_{j}}}$, for all $1 \leq j \leq k$ and $\alpha_{i_{1}}, \ldots, \alpha_{i_{k}}$ are some positive integers such that

$$
\alpha_{i_{1}} c_{i_{1}}+\cdots+\alpha_{i_{k}} c_{i_{k}} \equiv 1 \bmod m_{i_{1}} \cdots m_{i_{k}}
$$

In this case we obtain

$$
x^{d} \bmod N=\prod_{j=1}^{k} x^{\alpha_{i j} c_{i j} I_{i_{j}}} \bmod N .
$$

Thus, if an authorized group of users want to cooperate in computing $x^{d} \bmod N$, they individually compute the partial results of form $y_{i_{j}}=x^{\alpha_{i_{j}} c_{i_{j}} I_{i_{j}}} \bmod N$ and send them to a combiner who will compute the final result as

$$
\prod_{j=1}^{k} y_{i_{j}} \bmod N \text {. }
$$

In this way the private parameter will not be revealed to the members of the group or to the combiner.

\section{CONCLUSIONS}

We have surveyed the most important algorithms for the general Chinese remainder theorem. In our knowledge, such a survey does not exist in the literature. We have implemented and compared Ore's and Fraenkel's algorithm. Our tests showed that Fraenkel's algorithm behaves better in general, but in the case of modules known in advance, Ore's algorithm proves to be faster, thanks to precomputation enhancements. Moreover, we pointed out some interesting applications of this variant in secret sharing and threshold cryptography.

\section{REFERENCES}

[1] C. Ding, D. Pei, and A. Salomaa. Chinese remainder theorem: applications in computing, coding, cryptography. World Scientific Publishing Co., Inc., 1996.
[2] J.-J. Quisquater and C. Couvreur. Fast decipherment algorithm for the RSA public-key cryptosystem. IEE Electronics Letters 8(21) (1982), pp. 905-907.

[3] S. C. Pohlig and M. E. Hellman. An improved algorithm for computing logarithms over $G F(p)$ and its cryptographic significance. IEEE Transactions on Information Theory, 24:106110, 1978.

[4] M. Mignotte. How to share a secret. In T. Beth, editor, Proceedings of the Workshop on Cryptography, Burg Feuerstein, 1982, volume 149 of Lecture Notes in Computer Science, pages 371-375. Springer-Verlag, 1983.

[5] C. A. Asmuth and J. Bloom. A modular approach to key safeguarding. IEEE Transactions on Information Theory, IT29(2):208-210, 1983.

[6] H. Cohen. A Course in Computational Algebraic Number Theory. Graduate Texts in Mathematics. Springer-Verlag, 4th edition, 2000.

[7] F.L. Tiplea, S. Iftene, C. Hritcu, I. Goriac, R.M. Gordan and E. Erbiceanu. MpNT: A MultiPrecision Number Theory Package. NumberTheoretic Algorithms (I). Technical Report TR03-02 (2003), Faculty of Computer Science "A1.I.Cuza" University of Iasi. (http://www.infoiasi.ro/ tr/tr.pl.cgi)

[8] O. Ore. The general Chinese remainder theorem. American Mathematical Monthly, 59:365-370, 1952.

[9] C.F. Gauss, Disquisitiones Arithmeticae, 1801. English translation by Arthur A. Clarke, Springer-Verlag, New York, 1986.

[10]A. S. Fraenkel. New proof of the generalized Chinese remainder theorem. Proceedings of American Mathematical Society, 14:790-791, 1963.

[11]H. Garner. The residue number system. IRE Transactions on Electronic Computers EC-8 (1959), pp. 140-147.

[12]Y. Desmedt. Some recent research aspects of threshold cryptography. In E. Okamoto, G. I. Davida, and M. Mambo, editors, ISW '97: Proceedings of the First International Workshop on Information Security, volume 1396 of Lecture Notes in Computer Science, pages 158173. Springer-Verlag, 1998.

[13]R. Cramer, M. K. Franklin, B. Schoenmakers, and M. Yung. Multi-authority secret-ballot elections with linear work. In U. Maurer, editor, Advances in Cryptology - EuroCrypt '96, volume 1070 of Lecture Notes in Computer Science, pages 72-83. Springer-Verlag, 1996.

[14]A. Shamir. How to share a secret. Communications of the ACM, 22(11):612-613, 
1979.

[15]G. R. Blakley. Safeguarding cryptographic keys. In National Computer Conference, 1979, volume 48 of American Federation of Information Processing Societies Proceedings, pages 313-317, 1979.

[16]M. Ito, A. Saito, and T. Nishizeki. Secret sharing scheme realizing general access structure. In Proceedings of the IEEE Global Telecommunications Conference, Globecom '87, pages 99-102. IEEE Press, 1987

[17]J. Benaloh and J. Leichter. Generalized secret sharing and monotone functions. In $\mathrm{S}$. Goldwasser, editor, Advanced in CryptologyCRYPTO '88, volume 403 of Lecture Notes in Computer Science, pages 27-35. SpringerVerlag, 1989

[18]S. Iftene. A generalization of Mignotte's secret sharing scheme. In T. Jebelean, V. Negru, D. Petcu, and D. Zaharie, editors, Proceedings of the 6th International Symposium on Symbolic and Numeric Algorithms for Scientific Computing, Timisoara, Romania, September, 2004, pages 196-201, 2004.

[19]Y. Desmedt, G. Di Crescenzo, and M. Burmester. Multiplicative non-abelian sharing schemes and their applications to threshold cryptography. In J. Pieprzyk and R. SafaviNaini, editors, Advances in Cryptology Asiacrypt '94, volume 917 of Lecture Notes in Computer Science, pages 21-32. SpringerVerlag, 1995.
[20]S. Iftene. Threshold RSA Based on the General Chinese Remainder Theorem. Technical Report TR05-05 (2005), Faculty of Computer Science "Al.I.Cuza" University of Iasi. (http://www.infoiasi.ro/ tr/tr.pl.cgi)

[21]R. L. Rivest, A. Shamir and L. M. Adelman. $A$ Method for Obtaining Digital Signatures and Public-key Cryptosystems. Communications of the ACM 2 (21) (1978), pp. 120-126.

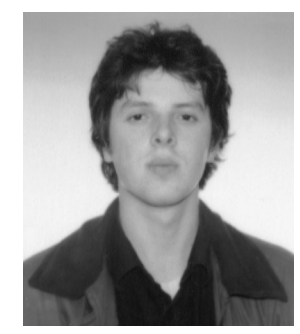

Sorin Iftene has received his M.Sc., B.Sc., and Ph.D. degrees at Faculty of Computer Science, "Al. I. Cuza" University, lasi, Romania, and is currently lecturer at the same faculty. $\mathrm{He}$ is interested in algebraic foundations of computer science, cryptography and computer security. His research was partially supported by the National University Research Council of Romania under the grant CNCSIS632/2006.

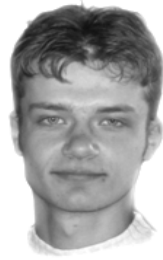

Florin Chelaru is a third-year student at Faculty of Computer Science, "Al. I. Cuza" University, lasi, Romania. He is interested in algebraic foundations of computer science, cryptography, data structures, algorithms and computer programming. 